\title{
Escala de Comportamiento Creativo: aportes metodológicos a los resultados de Aranguren e Irrazabal (2012)
}

\section{Creative Behavior Scale: methodological issues concerning Aranguren \& Irrazabal's (2012) results}

Palabras clave: análisis factorial exploratorio, consistencia interna, unidimensionalidad

Keywords: exploratory factor analysis, internal consistency, unidimensionality

En la presente carta se tiene como objetivo aportar en aspectos referidos a la metodología utilizada en el estudio de Aranguren e Irrazabal (2012), respecto a las propiedades de la Escala de Comportamiento Creativo (ECC) aplicada en Argentina a 258 sujetos adultos y a la consideración que se realizó sobre la validez de constructo y su aplicación en pruebas multidimensionales. Los dos puntos considerados son de carácter metodológico: el análisis del poder discriminante de los ítems que componen la escala y el análisis de la consistencia interna de la misma.

Respecto al primer punto, la información presentada arroja ciertas dudas sobre cómo fueron estimados los índices de discriminación de cada ítem y sobre el criterio empleado para considerar cuándo un índice es alto o bajo. El manuscrito dice "se calcularon las medias y las varianzas de cada uno de los sujetos comprendidos en el cuartil inferior y superior y se aplicó la prueba $t$ de Student de diferencia de medias". Entiendo que se refiere al poder discriminante $D$ basado en proporciones e interpreto que se calcularon las medias de las puntuaciones de cada dimensión para los cuartiles superior e inferior y que luego se aplicó el contraste $t$ de Student para cada uno de los ítems que componen cada dimensión. Este contraste no sería el procedimiento apropiado. La hipótesis nula de la prueba $t$ postula igualdad de medias de una variable (en este caso la puntuación en el ítem) en dos muestras extraídas aleatoriamente. Pero en este caso las muestras (cuartiles superior e inferior) derivan de un procedimiento que maximiza una diferencia (la puntuación en la dimensión), la cual a su vez contiene a la diferencia que el estadístico de contraste pretende evaluar (la puntuación en el ítem). Si se desea evaluar el poder discriminante $D$ se debe calcular la diferencia entre las puntuaciones medias del ítem en ambos grupos dividida entre el número de casos y remitirse a los valores de referencia de $D$ empleados comúnmente (por ejemplo, Ebel, 1979).

Respecto al segundo punto, la fiabilidad de una dimensión o factor puede estimarse a partir de la consistencia interna de Cronbach (1951), entre otros indicadores. No obstante, un requisito necesario para una correcta interpretación del coeficiente a es el de unidimensionalidad. Cuando un cuestionario es unidimensional el $\alpha$ de Cronbach representa el valor medio de todos los posibles coeficientes de correlación entre las dos mitades en que puede dividirse el cuestionario. Así, a constituye una medida de la proporción de varianza compartida por los ítems que se nuclean en torno a una misma dimensión. De acuerdo a los resultados presentados, la ECC tendría cuatro dimensiones, por lo que solamente correspondería informar los valores de a para cada subescala, pero no un a global. Puesto que las autoras emplearon el método Varimax para rotar los factores extraídos estas dimensiones necesariamente resultan ortogonales. Sería conveniente repetir los análisis empleando métodos de rotación oblicua; las correlaciones así obtenidas podrían dar un indicio de si efectivamente se trata de un modelo de cuatro factores independientes (correlaciones bajas) o si podría tratarse de cuatro dimensiones nucleadas en torno a un factor de segundo orden (correlaciones moderadas o altas). En este último caso tendría sentido estimar un a global, previo ajuste de un modelo de segundo orden mediante un análisis factorial confirmatorio.

Puesto que en el trabajo no se justifica la estimación de un a global, este constituye un aspecto metodológico importante. Ello podría desorientar a un lector no familiarizado con estas técnicas, llevándolo a concluir erróneamente que un valor de a global elevado es sinónimo 
de validez de un constructo global, cuando en realidad a no arroja información sobre la estructura factorial subyacente a los datos (Campbell, 1960; Schmitt, 1996; Sijtsma, 2009).

Lo anterior también cuestiona el empleo de la puntuación total en la ECC para evaluar la validez convergente y la validez de criterio, así como la asociación con variables sociodemográficas. Es decir, las autoras no presentan evidencia de la existencia de un constructo "comportamiento creativo". Sin embargo, dicha conceptualización se vincula con otros constructos (personalidad creativa y autoeficacia creativa) y se comentan las diferencias de comportamiento creativo entre hombres y mujeres, así como entre adultos jóvenes y adultos medios.

Es importante tener en cuenta los aspectos mencionados a fin de garantizar los resultados, a sabiendas del esfuerzo que suponen este tipo de estudios y la posibilidad que dan de establecer este tipo de discusiones metodológicas y de aportar de otros investigadores, alegrándonos de que exista la posibilidad y de que ésta inicie un diálogo fecundo

María Noel Rodríguez Ayán
Unidad Académica de Educación Química
Facultad de Química
Universidad de la República
Isidoro de María 1620, $2^{\circ}$ piso CP 11800 Montevideo
Tel/Fax: $598-2929-0770$
mail: mayan@fq.edu.uy

\section{Referencias}

Aranguren, M. e Irrazabal, N. (2012). Diseño de una escala para la evaluación del comportamiento creativo en diferentes dominios. Ciencias Psicológicas, VI(1), 29-41.

Campbell, D.T. (1960). Recommendations for APA tests regarding construct, trait or discriminant validity. American Psychologist, 15, 546-553.

Cronbach, L. J. (1951). Coefficient alpha and the internal structure of tests. Psychometrika, 16(3), 297-334.

Ebel, R. L. (1979). Essentials of Educational Measurement. Englewood Cliffs, New Jersey: Prentice Hall Inc.

Schmitt, N. (1996). Uses and abuses of coefficient alpha. Psychological Assessment, 8(4), 350-353.

Sijtsma, K. (2009). On the use, the misuse, and the very limited usefulness of Cronbach's alpha. Psychometrika, 74(1), 107-120. 